\title{
The importance of gender difference in evaluation of relationship between MPV and serum biochemical parameters in type 2 diabetic patients
}

\section{Yildiz Okuturlar, Hakan Kocoglu, Omer Seker, Betul Erismis, Bahar Ozdemir, Meral Mert, Sibel Ocak Serin*, Mehmet Hursitoglu, Ozlem Harmankaya, Abdulbaki Kumbasar}

Bakirkoy Dr. Sadi Konuk Education and Research Hospital

*Umraniye Education and Research Hospital, Istanbul

\section{Objectives:}

Increased mean platelet volume (MPV) is emerging as an independent risk factor for thromboembolism, stroke and myocardial infarction. But the importance of gender difference in evaluation MPV levels is not known. Thus, in this study we tried to emphasize the importance of gender difference in evaluation of relationship between MPV and serum biochemical parameters in type 2 diabetic patients.
In this retrospective study, a total of 509 patients with diabetes mellitus who hospitalized due to several causes were evaluated. Their age, gender, other comorbidities, $\mathrm{HbAlc}$, and other biochemical parameters were recorded. All data were analyzed by using SPSS 22.0 statistical package for Windows.

\section{Results:}

A total of 509 patients consisted of 269 women and 240 men were included in this study. There were no significantly difference in regard to other comorbidities between genders $(\mathrm{p}>0.05)$. Mean age of women was $65.43 \pm 16.1$ years and the mean age of men was $64.84 \pm 14.98$ years $(\mathrm{p}=0.669)$. The mean MPV value of women was $8.61 \pm 1.40 \mathrm{fL}$ and the mean MPV value of men was $8.52 \pm 1.47 \mathrm{fL}$. MPV value did not differ according to gender $(p=0.486)$. Erythrocyte sedimentation rate $(E S R)(p=0.016)$, HDL-cholesterol $(\mathrm{Chol})(\mathrm{p}=0.024)$, total-Chol $(\mathrm{p}=0.01)$, and platelet $(\mathrm{PLT})$ $(p=0.035)$ levels were significantly higher in women whereas urea $(p=0.08)$, creatinine $(p=0.0001)$, and triglyceride $(p=0.002)$ levels were significantly higher in men (Table 1). There were positive correlations between MPV and urea, creatinine, uric acid, and PDW values and there were negative correlations between MPV and total-chol, LDL-chol, and PLT in women. Also there were positive correlations between MPV and HbAlc, glucose, uric acid, and PDW values and there were negative correlations between MPV and ESR and PLT values in men.

\begin{tabular}{|c|c|c|c|c|c|c|c|}
\hline \multicolumn{4}{|c|}{ Male Patients ( $n=240$ ) } & \multicolumn{3}{|c|}{ Female Patients $(n=269)$} & \multirow{2}{*}{$p$ value } \\
\hline Variable & Minimum & Maximum & Mean \pm SD & Minimum & Maximum & Mean $\pm S D$ & \\
\hline Age (year) & 20 & 95 & $64.84 \pm 14.98$ & 20 & 96 & $65.43 \pm 16.10$ & 0.669 \\
\hline ESR (mm/hr) & 1 & 120 & $47.90 \pm 35.5$ & 2 & 120 & $55.63 \pm 33.43$ & 0.016 \\
\hline HbA1c (\%) & 4.60 & 16.80 & $7.62 \pm 2.58$ & 4.60 & 17.60 & $7.98 \pm 2.75$ & 0.162 \\
\hline Glucose (mg/dL) & 18 & 886 & $195.29 \pm 159.54$ & 15 & 1455 & $222.03 \pm 196.97$ & 0.086 \\
\hline Urea (mg/dL) & 6 & 689 & $110.68 \pm 86.71$ & 15 & 433 & $92.52 \pm 70.64$ & 0.008 \\
\hline Creatinine (mg/dL) & 0.30 & 146.50 & $3.85 \pm 9.80$ & 0.34 & 15.98 & $2.45 \pm 2.45$ & 0.0001 \\
\hline Uric acid (mg/dL) & 1.80 & 20.90 & $7.15 \pm 2.69$ & 1.30 & 19.81 & $7.35 \pm 3.18$ & 0.446 \\
\hline HDL-Chol (mg/dL) & 5 & 79 & $33.77 \pm 12.63$ & 5 & 90 & $37.21 \pm 15.03$ & 0.024 \\
\hline Total-Chol (mg/dL) & 52 & 470 & $160.70 \pm 60.25$ & 72 & 354 & $174.97 \pm 55.67$ & 0.001 \\
\hline LDL-Chol (mg/dL) & 2 & 336 & $95.13 \pm 45.58$ & 13 & 247 & $99.95 \pm 40.78$ & 0.107 \\
\hline Triglycerid (mg/dL) & 24 & 7115 & $228.28 \pm 674.31$ & 42 & 1375 & $190.93 \pm 161.16$ & 0.002 \\
\hline CRP (mg/L) & 0.03 & 46.58 & $6.60 \pm 8.93$ & 0.02 & 32 & $5.04 \pm 6.70$ & 0.476 \\
\hline WBC x103 (/mm3) & 0.7 & 63.9 & $10.53 \pm 6.61$ & 1.2 & 37.5 & $10.38 \pm 6.32$ & 0.440 \\
\hline PLT x103 (/mm3) & 5 & 685 & $252.7 \pm 125.1$ & 3 & 1118 & $277.9 \pm 139.7$ & 0.035 \\
\hline PDW (\%) & 11.30 & 34.80 & $17.48 \pm 2.77$ & 9.30 & 27.50 & $17.34 \pm 2.36$ & 0.836 \\
\hline MPV (fL) & 5.33 & 17.10 & $8.52 \pm 1.47$ & 5.03 & 15.10 & $8.61 \pm 1.39$ & 0.486 \\
\hline
\end{tabular}

Table 1. difference of patients' characteristics and blood parameters among genders.

SD: standard deviation, ESR: erythrocyte sedimentation rate, $\mathrm{HbAlc}$ hemoglobin alc, -chol: cholesterol, CRP: c-reactive protein, WBC white blood cell, PLT: platelet, PDW: platelet distribution width, MPV: mean platelet volume. A value of $P<0.05$ was considered statistically significant.

\section{Conclusion:}

We did not find any difference in MPV values between male and female patients with diabetes. But we have showed that the significance of correlation analysis between MPV and each biochemical parameter depended upon gender. Thus the importance of gender difference in studies evaluating MPV levels in diabetic patients should be considered. 